\title{
On robust mean-variance portfolios
}

\section{Mustafa Ç. Pınar}

To cite this article: Mustafa Ç. Pınar (2016) On robust mean-variance portfolios, Optimization, 65:5, 1039-1048, DOI: 10.1080/02331934.2015.1132216

To link to this article: https://doi.org/10.1080/02331934.2015.1132216

$$
\text { 曲 Published online: 08 Jan } 2016 .
$$

Submit your article to this journal

\section{Шll Article views: 247}

\section{Q View related articles $\sqsubset$}

View Crossmark data $\nearrow$

47 Citing articles: 6 View citing articles 진 


\title{
On robust mean-variance portfolios
}

\author{
Mustafa Ç. Pınar \\ Department of Industrial Engineering, Bilkent University, Bilkent, Ankara, Turkey
}

\begin{abstract}
We derive closed-form portfolio rules for robust mean-variance portfolio optimization where the return vector is uncertain or the mean return vector is subject to estimation errors, both uncertainties being confined to an ellipsoidal uncertainty set. We consider different mean-variance formulations allowing short sales, and derive closed-form optimal portfolio rules in static and dynamic settings.
\end{abstract}

\section{ARTICLE HISTORY}

Received 8 October 2014

Accepted 9 December 2015

\section{KEYWORDS}

Robust optimization; mean-variance portfolio theory; ellipsoidal uncertainty; adjustable robustness

\section{Introduction}

The problem of an investor seeking to minimize the variance of the return of his/her portfolio while maintaining a minimum expected target return is a fundamental problem of the mean-variance (MV) portfolio theory introduced by Markowitz [1]. Alternatively, one can try to maximize expected return while maintaining a cap on portfolio return variance. The problem is at the root of modern finance, and has become an integral part of the majority of textbooks in finance; see e.g. [2-4]. For a more recent survey on MV portfolio selection the reader is referred to [5]. Allowing for short positions in the optimal portfolio and excluding other portfolio restrictions, it is a quadratic programming problem that can be solved explicitly. In this brief paper, we extend this simple yet fundamental result to the case where the estimation error in the mean return vector is taken into account using the ellipsoidal robustness paradigm [6-10] and we deal with optimization problems containing second-order cone elements. Since the true mean returns are never known exactly, one has to deal with estimated mean returns, and the negative effect of imprecision of mean returns on optimal portfolios has been studied and documented in e.g. [11-13] (the effect of imprecision in variance/covariance is known to be less pronounced). The imprecision in mean return has been addressed in portfolio optimization using conic optimization and numerical algorithms. $[9,14-20]$ The purpose of this paper is to investigate analytical solutions in the absence of short sales and other portfolio restrictions such as different rates for borrowing and lending, transaction costs and so on. The static explicit portfolio rules and an assumption of independent returns between consecutive periods enable us to extend our results to the case of multi-period dynamic MV portfolio policies under uncertainty in the mean returns using the adjustable robustness criterion.[21-23] We also consider robust portfolios where the return vector of risky assets is subject to ellipsoidal uncertainty, rather than the mean return vector being uncertain. We give closed-form portfolio rules and investigate dynamic adjustable robust portfolio selection in that case. The dynamic portfolio rules turn out to be myopic policies. To the best of our knowledge, the simple closed-form portfolio rules of the present paper were not published previously in the literature since most authors (except [18]) concentrated on the numerical solution of robust portfolio problems by modern solvers, and did not consider the relatively simpler cases treated in the present paper. The absence of attention to the models treated in the present paper is a gap to be 
filled since most expositions to the theory of Markowitz MV efficient portfolios begin by allowing short sales and unlimited borrowing to lead to the most fundamental insights of the theory (see e.g. $[2,5])$. It is our hope in circulating the present paper that the results given here will kindle an interest in the portfolio optimization community to re-study basic models using the closed-form rules of the present paper.

\section{The static robust MV portfolios}

Consider the problem of a MV investor operating in a market with $m$ risky assets and a riskless asset with period return equal to $R$. The risky assets have mean return $r$ that is assumed unknown, and variance-covariance matrix $\Sigma$, assumed positive definite. The investor is assumed to have an initial wealth equal to $W_{0}$. Under the supposition that the expected risky asset returns $r$ are uncertain, we shall confine this uncertainty into the ellipsoidal uncertainty set

$$
U_{r}=\left\{r:\left\|\Sigma^{1 / 2}(r-\hat{r})\right\|_{2} \leq \gamma\right\}
$$

where $\gamma$ is a positive scalar and $\hat{r}$ is an estimate of mean return vector. For a target wealth $T$, we shall examine the following 'robust' MV portfolio choice problem

$$
\min _{x} \frac{1}{2} x^{T} \Sigma x
$$

subject to

$$
r^{T} x+\left(W_{0}-e^{T} x\right) R \geq T \quad \forall r \in U_{r}
$$

where we use $e$ to represent an $m$-vector of ones. It is well known that the above semi-infinite problem is equivalent to the following convex optimization problem with a second-order cone constraint (see e.g. [6,7]), referred to as RMVP1 (short for robust mean-variance problem 1):

$$
\min _{x} \frac{1}{2} x^{T} \Sigma x
$$

subject to

$$
\hat{r}^{T} x+\left(W_{0}-e^{T} x\right) R-\gamma\left\|\Sigma^{1 / 2} x\right\|_{2} \geq T .
$$

We shall make the blanket assumption that $T>W_{0} R$. Otherwise, it is optimal to invest all wealth into the riskless asset. The problem above has been studied in different forms numerically for the case where short sales are not allowed. However, a study of the problem with short positions allowed is, to the best of our knowledge, still missing in the literature. The purpose of this note is to fill this gap. In our proofs we use an ansatz inspired from [18 $]^{1}$ and based on assuming a non-zero optimal point, then working out a specific formula, and figuring out the parameters of the formula.

We define the excess mean return vector estimate $\hat{\mu}=\hat{r}-R e$ and the square root of the optimal Sharpe ratio in the market $H=\sqrt{\hat{\mu}^{T} \Sigma^{-1} \hat{\mu}}$ (the slope of the capital market line).

Proposition 1: If RMVP1 satisfies the Slater condition (there exists $\hat{x}$ such that $\hat{r}^{T} \hat{x}+\left(W_{0}-e^{T} \hat{x}\right) R-$ $\left.\gamma\left\|\Sigma^{1 / 2} \hat{x}\right\|_{2}>T\right)$ and $H>\gamma$, then RMVP1 admits the unique optimal solution

$$
x^{*}=\left(\frac{T-W_{0} R}{H(H-\gamma)}\right) \Sigma^{-1} \hat{\mu} .
$$

If $H<\gamma$, then there is no feasible portfolio in RMVP1 while for $H=\gamma$ the optimal portfolio takes infinite holdings in all risky assets. 
Proof: The problem is convex. We write the Lagrange function using a non-negative multiplier $\lambda$ as follows:

$$
L(x, \lambda)=\frac{1}{2} x^{T} \Sigma x+\lambda\left(T-\hat{\mu}^{T} x-W_{0} R+\gamma\left\|\Sigma^{1 / 2} x\right\|_{2}\right) .
$$

The first-order conditions (that are necessary and sufficient under Slater constraint qualification, see [8]) give

$$
\Sigma x-\lambda \hat{\mu}+\lambda \gamma \frac{\Sigma x}{\sqrt{x^{T} \Sigma x}}=0 .
$$

Let us define $\sigma=\sqrt{x^{T} \sum x}$ and solve for $x$ from the first-order conditions (assuming a solution $x \neq 0$ ). This yields $x^{*}=\frac{\sigma \lambda}{\sigma+\lambda \gamma} \Sigma^{-1} \hat{\mu}$. By definition of $\sigma$, substituting $x$ we have the identity $\sigma^{2}=\frac{\sigma^{2} \lambda^{2}}{(\sigma+\lambda \gamma)^{2}} H^{2}$, which implies that $H^{2}=\frac{(\sigma+\lambda \gamma)^{2}}{\lambda^{2}}$, or, equivalently by positivity of all $\sigma, \gamma$ and $H$, $H=\frac{\sigma+\lambda \gamma}{\lambda}$. Observing that $\hat{\mu}^{T} x^{*}=\frac{\sigma \lambda}{\sigma+\lambda \gamma} H^{2}$ and supposing that the constraint is binding we have $\frac{\sigma \lambda}{\sigma+\lambda \gamma} H^{2}+W_{0} R-\gamma \frac{\sigma \lambda}{\sigma+\lambda \gamma} H=T$. Simplifying we get $\sigma H+W_{0} R-\gamma \sigma=T$. Hence $\sigma=\frac{T-W_{0} R}{H-\gamma}$ from which we have the condition $\gamma<H$ for positivity of $\sigma$. Solving for $\lambda$ from $H=\frac{\sigma+\lambda \gamma}{\lambda}$ we obtain $\lambda=\frac{\left(T-W_{0} R\right)}{(H-\gamma)^{2}}$. Now, substituting $\sigma$ and $\lambda$ into the expression for $x^{*}$ we obtain the announced result. If $H<\gamma$, we have a $\sigma<0$, which implies that no feasible portfolio exists.

Notice that for $\gamma=0$ we recover the classical MV portfolio,

$$
x_{m v}=\frac{T-W_{0} R}{H^{2}} \Sigma^{-1} \hat{\mu} .
$$

Here, an interesting extension would be to disallow borrowing by introducing the constraint $W_{0}-$ $e^{T} x \geq 0$ into the model. Unfortunately, the presence of this constraint renders the analytical solution of the KKT system in the above proof impossible since it results in a fourth-degree polynomial. One can easily see that the result will be of the form:

$$
x^{*}=A \Sigma^{-1} \hat{\mu}+B \Sigma^{-1} e,
$$

where $A$ and $B$ are problem-dependent constants to be determined numerically.

As an alternative to the above model, one can start with the following robust MV model

$$
\max _{x} \min _{r \in U_{r}} r^{T} x+\left(W_{0}-e^{T} x\right) R
$$

subject to

$$
x^{T} \Sigma x \leq t^{2}
$$

for an appropriately chosen positive real number $t$. This model is equivalent to

$$
\max _{x} \hat{r}^{T} x+\left(W_{0}-e^{T} x\right) R-\gamma\left\|\Sigma^{1 / 2} x\right\|_{2}
$$

subject to

$$
x^{T} \Sigma x \leq t^{2}
$$

or, equivalently

$$
\max _{x} \hat{\mu}^{T} x+W_{0} R-\gamma\left\|\Sigma^{1 / 2} x\right\|_{2}
$$

subject to

$$
x^{T} \Sigma x \leq t^{2} .
$$

We shall refer to the above problem as RMVP2. 
Proposition 2: If $H \geq \gamma$ then RMVP2 admits the unique optimal solution

$$
x^{*}=\frac{t}{H} \Sigma^{-1} \hat{\mu} .
$$

If $H<\gamma$ then it is optimal for an RMVP2 investor to keep all initial wealth in the riskless asset.

Proof: Writing the Lagrange function using a non-negative multiplier $\lambda: L(x, \lambda)=\hat{\mu}^{T} x+W_{0} R-$ $\gamma\left\|\Sigma^{1 / 2} x\right\|_{2}+\lambda\left(t^{2}-x^{T} \Sigma x\right)$. The first-order conditions (necessary and sufficient since the problem is convex, and Slater condition holds trivially provided that $\left.t^{2}>0\right)$ yields $x^{*}=\left(\frac{\sigma}{2 \sigma \lambda+\gamma}\right) \Sigma^{-1} \hat{\mu}$. By definition of $\sigma$, we have $\sigma^{2}=\frac{\sigma^{2}}{(2 \sigma \lambda+\gamma)^{2}} H^{2}$. Assuming the constraint to be active and observing that $\sigma=t$, we solve for $\lambda$ and obtain: $\lambda=\frac{H-\gamma}{2 t}$, which is non-negative provided that $H \geq \gamma$. If $H<\gamma$ then the only feasible choice for $\lambda$ is zero along with a dual objective function value equal to $W_{0} R$ which is attained in the primal by a riskless portfolio, i.e. $x^{*}=0$.

Interestingly, we observe that the robust MV portfolio is identical to the MV portfolio obtained as a solution to the problem

$$
\max _{x} \hat{\mu}^{T} x+W_{0} R
$$

subject to

$$
x^{T} \Sigma x \leq t^{2}
$$

in the range $0 \leq \gamma \leq H$. That is to say, the investor maximizing robust expected return under a variance constraint makes a MV portfolio choice when his/her confidence in the estimate of the mean is high, i.e. $\gamma$ is smaller than the optimal Sharpe ratio $H$ of the market.

Corollary 1: Let $H>\gamma$. Then

(1) choosing a maximum variance $t=\frac{T-W_{0} R}{(H-\gamma)}$ the RMVP2 investor holds an optimal portfolio identical to the RMVP1 investor with a target wealth equal to $T$,

(2) choosing a minimum target wealth equal to $T=W_{0} R+t(H-\gamma)$ the RMVP1 investor holds an optimal portfolio identical to the RMVP2 investor with a variance cap equal to $t^{2}$.

In the next section, we shall see that using RMVP2 we can also solve in a very simple fashion the multiple period version of the robust MV portfolio problem in the setting of adjustable robustness.

There is yet another MV portfolio selection model that can be treated with robust optimization and yields an explicit portfolio rule. Consider the following problem

$$
\max _{x} \min _{r \in U_{r}} r^{T} x+\left(W_{0}-e^{T} x\right) R-\frac{\rho}{2} x^{T} \Sigma x
$$

where $\rho$ is a positive scalar. Processing the inner min we obtain as usual the problem:

$$
\max _{x} \hat{r}^{T} x+\left(W_{0}-e^{T} x\right) R-\gamma\left\|\Sigma^{1 / 2} x\right\|_{2}-\frac{\rho}{2} x^{T} \Sigma x
$$

that is referred to as RMVP3.

Proposition 3: If $\gamma \rho<H$ then RMVP3 admits the unique optimal solution

$$
x^{*}=\left(\frac{H-\gamma \rho}{\rho H}\right) \Sigma^{-1} \hat{\mu} .
$$

If $\gamma \rho>H$ then it is optimal for an RMVP3 investor to keep all initial wealth in the riskless asset. 
Proof: The function is strictly concave. The first-order necessary and sufficient conditions (assuming a solution $x \neq 0$ ) yield the candidate solution:

$$
x=\left(\frac{\sigma}{\gamma+\sigma \rho}\right) \Sigma^{-1} \hat{\mu} .
$$

Going through the usual steps, we have $H^{2}=(\gamma+\sigma \rho)^{2}$. Developing the right-hand side, we obtain a quadratic equation $\rho^{2} \sigma^{2}+2 \gamma \rho \sigma+\gamma^{2}-H^{2}=0$ with the positive root $\sigma_{+}=\frac{H-\gamma \rho}{\rho^{2}}$ provided that $\gamma \rho<H$. Then, the result follows by simple algebra.

One can, in the style of Corollary 1, identify parameter choices that ensure passage from the previous problems RMVP1 and RMVP2 to RMVP3 and vice versa. This is left as an exercise.

\section{The dynamic robust MV portfolios}

In this section, we shall obtain a solution to the multi-period portfolio selection problem in the case RMVP2 using the framework of adjustable robust optimization for the setting given as follows. For simplicity of exposition, let us develop the result for a two-period problem. There are two time points $n=0,1$ at which the portfolio choice is made, the end of the time horizon $n=2$ (in general $n=N)$ is the moment where the final realized portfolio value, say $W_{2}\left(W_{N}\right)$ is revealed. The $m$ risky assets have independent uncertain mean returns in each time period, i.e. the mean return vector $r_{1}$ is uncertain around the estimate $\hat{r}_{1}$ with positive definite variance-covariance matrix $\Sigma_{1}$, and similar statements hold for mean vector $r_{2}$ with estimate $\hat{r}_{2}$ and positive definite variance-covariance matrix $\Sigma_{2}$. For simplicity, the riskless asset is assumed to have invariant per period return $R \geq 1$. In case the riskless rates of return differ between periods, we shall assume that they are known a priori, a standard assumption. We confine the mean return $r_{1}$ of the first period to take values in the ellipsoidal ambiguity set around $\hat{r}_{1}: U_{r}^{1}=\left\{r_{1} \mid\left\|\Sigma_{1}^{-1 / 2}\left(r_{1}-\hat{r}_{1}\right)\right\|_{2} \leq \gamma_{1}\right\}$, and the mean return $r_{2}$ of the second period to take values in the ellipsoidal ambiguity set around $\hat{r}_{2}: U_{r}^{2}=\left\{r_{2} \mid\left\|\Sigma_{2}^{-1 / 2}\left(r_{2}-\hat{r}_{2}\right)\right\|_{2} \leq \gamma_{2}\right\}$.

The adjustable robustness framework we propose consists in making the dynamic portfolio choice according to the solution of the following problem:

$$
V_{2}=\max _{x_{2} \in X_{2}} \min _{r_{2} \in U_{r}^{2}} r_{2}^{T} x_{2}+\left(W_{1}-e^{T} x_{2}\right) R
$$

and

$$
V_{1}=\max _{x_{1} \in X_{1}} \min _{r_{1} \in U_{r}^{1}} V_{2}
$$

where $X_{1}=\left\{x \in \mathbb{R}^{m} \mid x^{T} \Sigma_{1} x \leq t_{1}^{2}\right\}$ and $X_{2}=\left\{x \in \mathbb{R}^{m} \mid x^{T} \Sigma_{2} x \leq t_{2}^{2}\right\}$ for positive constants $t_{1}$ and $t_{2}$. We use $W_{1}$ to represent the portfolio value at the end of period 1, i.e. at time point $n=1$ where the investor is about to make the second-period portfolio decision. Obviously, for an observer at time point $n=0$, both $W_{1}$ and $W_{2}$ are uncertain quantities. However, the observer at time $n=1$ will have already observed $W_{1}$; therefore, at the moment of making the choice for $x_{2}$ (i.e. beginning of stage 2) $W_{1}$ is no longer uncertain. We shall exploit this observation in solving the problem. The resulting optimal portfolio policy is therefore non-anticipative. It is usually not expected to solve the adjustable robust dynamic portfolio problems analytically. However, as we shall see, the case studied here constitutes an exception, due to the assumption of independence of returns between consecutive periods.

We solve the problem backwards, i.e. starting from stage 2. At the beginning of stage 2 , the endof-stage 1 wealth $W_{1}$ is a known quantity that the investor is aiming to distribute optimally among the $m+1$ assets. Therefore, the following problem is solved:

$$
\max _{x_{2} \in X_{2}} \hat{r}_{2}^{T} x_{2}+\left(W_{1}-e^{T} x_{2}\right) R-\gamma_{2}\left\|\Sigma_{2}^{1 / 2} x_{2}\right\|_{2}
$$


By Proposition 2, the solution $x_{2}^{*}$ is given by

$$
x_{2}^{*}=\frac{t_{2}}{H_{2}} \Sigma_{2}^{-1} \hat{\mu}_{2}
$$

where $H_{2}=\hat{\mu}_{2}^{T} \Sigma_{2}^{-1} \hat{\mu}_{2}$ and $\hat{\mu}_{2}=\hat{r}_{2}-R e$, provided that $H_{2} \geq \gamma_{2}$. This solution results in the expression $V_{2}=\kappa_{2}+R W_{1}$, where $\kappa_{2}=t_{2}\left(H_{2}-\gamma_{2}\right)$ is defined for simplifying the notation. Now, let us return to stage 1 (i.e. beginning of period $1, n=0$ ) with this solution. We have to address the problem of computing $V_{1}$ where $V_{1}=\max _{x_{1} \in X_{1}} \min _{r_{1} \in U_{r}^{1}} \kappa_{2}+R W_{1}$ with $W_{1}=x_{1}^{T} r_{1}+\left[W_{0}-e^{T} x_{1}\right] R$. Hence we need to solve the problem $\max _{x_{1} \in X_{1}} \kappa_{2}+R\left(\hat{\mu}_{1}^{T} x_{1}+W_{0} R-\gamma_{1}\left\|\Sigma^{1 / 2} x_{1}\right\|_{2}\right)$. One proceeds exactly as in the proof of Proposition 2 and obtains the unique optimal portfolio: $x_{1}^{*}=\frac{t_{1}}{H_{1}} \Sigma_{1}^{-1} \hat{\mu}_{1}$, provided that $H_{1} \geq \gamma_{1}$. The above process can be easily generalized for arbitrary integer number $N>2$ of periods. Hence, we can assert the following result. The sets $X_{3}, \ldots, X_{N}$ are defined analogously to $X_{1}, X_{2}$ with positive $t_{3}, \ldots, t_{N}$.

Proposition 4: The adjustable robust dynamic portfolio policy of an RMVP2 investor with uncertainty parameters $\gamma_{n}, n=1, \ldots, N$ and initial wealth $W_{0}$, defined as the solution to the following problem:

$$
\begin{gathered}
V_{N}=\max _{x_{N} \in X_{N}} \min _{r_{N} \in U_{r}^{N}} r_{N}^{T} x+\left(W_{N-1}-e^{T} x_{N}\right) R \\
V_{N-1}=\max _{x_{N-1} \in X_{N-1}} \min _{r_{N-1} \in U_{r}^{N-1}} V_{N} \\
\vdots \\
V_{1}=\max _{x_{1} \in X_{1}} \min _{r_{1} \in U_{r}^{1}} V_{2},
\end{gathered}
$$

is given by

$$
x_{n}^{*}=\frac{t_{n}}{H_{n}} \Sigma_{n}^{-1} \hat{\mu}_{n}, n=1, \ldots, N,
$$

where $\hat{\mu}_{n}=\hat{r}_{n}-R e$, provided that $\gamma_{n} \leq H_{n}$ for all $n=1, \ldots, N$. The values $V_{n}, n=1, \ldots, N$ are given by $V_{n}=\sum_{\ell=n}^{N} R^{N-\ell} \kappa_{\ell}+R^{N-n+1} W_{n-1}$, where $\kappa_{n}=t_{n}\left(H_{n}-\gamma_{n}\right)$.

It is a simple exercise to see that even with the interest rate changing from period to period, i.e. with $R_{n}, n=1, \ldots, N$, the optimal policy of Proposition 4 continues to hold unchanged. Notice that the optimal dynamic policy of Proposition 4 is a very simple myopic policy as it completely disregards the fact that the investor will have an opportunity to re-invest in subsequent periods; see [24] for a discussion of multi-period portfolio policies and [25] for a multi-period MV optimal policy based on dynamic programming. As a final remark, we note that with neither RMVP1 nor RMVP3 we were able to obtain an explicit adjustably robust policy as in Proposition 4. RMVP1 seems to give an intractable problem, whereas RMVP3 does not yield a finite policy (i.e. it leads to unbounded portfolio policies; c.f., end of the next section).

\section{The Ben-Tal and Nemirovski robust portfolios}

In a seminal paper [6] where they introduced ellipsoidal uncertainty sets and robust optimization concepts, Ben-Tal and Nemirovski studied a simple portfolio selection example which presents a departure from the MV portfolio model. They treated the return vector of risky assets as an uncertain quantity and confined it to an ellipsoidal set, instead of treating the mean return vector as an uncertain entity as we have done in the previous sections. We are dealing with $m$ risky assets. The return of risky assets, an uncertain quantity, is denoted $r$. The investor is assumed to have an initial wealth 
equal to $W_{0}$. Under the supposition that the risky asset returns $r$ are confined into the ellipsoidal uncertainty set

$$
U_{r}=\left\{r:\left\|\Sigma^{1 / 2}(r-\hat{r})\right\|_{2} \leq \epsilon\right\}
$$

where $\Sigma$ is a symmetric positive definite matrix (hence, it has a square root; $\Sigma$ can be taken as the estimate of the variance-covariance matrix if historical data are available), $\epsilon$ is a positive scalar representing the uncertainty radius and $\hat{r}$ is some estimate of return vector (referred to as the nominal return in [6]). The resulting robust model is as follows (BTNRP):

$$
\max _{x} \hat{r}^{T} x-\epsilon\left\|\Sigma^{1 / 2} x\right\|_{2}
$$

subject to

$$
e^{T} x=W_{0}
$$

The above model is not exactly identical to the Ben-Tal and Nemirovski model in that they do not allow short sales, thus have non-negativity restrictions, and work with a diagonal $\Sigma$ in their example. We have left out the riskless asset in the above model intentionally since its presence leads to extreme optimal portfolios as we shall see below.

We define the following quantities that are useful:

$$
A=\hat{r}^{T} \Sigma^{-1} \hat{r}, B=e^{T} \Sigma^{-1} \hat{r}, C=e^{T} \Sigma^{-1} e, \Delta=B^{2}-A C+C \epsilon^{2} .
$$

Proposition 5: If the uncertainty radius $\epsilon$ satisfies: $\epsilon>\sqrt{A-\frac{B^{2}}{C}}$ then the BTNRP investor makes the optimal portfolio choice:

$$
x^{*}=\frac{W_{0}}{\sqrt{\Delta}} \Sigma^{-1}\left(\hat{r}-\frac{B-\sqrt{\Delta}}{C} e\right) .
$$

If $\epsilon \leq \sqrt{A-\frac{B^{2}}{C}}$ then BTNRP is unbounded.

Proof: The problem is convex. Forming the Lagrange function using an unrestricted multiplier $\lambda$ :

$$
L(x, \lambda)=\hat{r}^{T} x-\epsilon\left\|\Sigma^{1 / 2} x\right\|_{2}+\lambda\left(W_{0}-e^{T} x\right),
$$

we obtain:

$$
x^{*}=\frac{\sigma}{\epsilon} \Sigma^{-1}(\hat{r}-\lambda e),
$$

where we assume a non-null optimal solution and defined as usual $\sigma=\sqrt{x^{T} \Sigma x}$. Using the previous definition of $\sigma$, we obtain the quadratic equation: $C \lambda^{2}-2 B \lambda+A-\epsilon^{2}=0$ with the roots $\frac{B \pm \sqrt{\Delta}}{C}$, which imposes the condition $\epsilon>\sqrt{A-\frac{B^{2}}{C}}$ to ensure positivity of $\Delta$ (notice that $A-B^{2} / C>0$ since we have $A C-B^{2}>0$ by Cauchy-Schwarz inequality and $C>0$ by positive definiteness of $\Sigma$ ). From the constraint equation, we have $\sigma=\frac{W_{0} \epsilon}{B-\lambda C}$. Since we need $\sigma>0$ we require $B-\lambda C>0$, which imposes the choice of the root with the negative sign in front of $\sqrt{\Delta}$. Thus, we have $\lambda^{*}=\frac{B-\sqrt{\Delta}}{C}$ and, after evident simplification we obtain $\sigma=\frac{W_{0} \epsilon}{\sqrt{\Delta}}$. Substituting these quantities into the candidate solution, we arrive at the announced result. If $\epsilon \leq \sqrt{A-\frac{B^{2}}{C}}$ then we have an infeasible Lagrange dual problem, which implies that the primal is unbounded (since the primal is trivially feasible).

When we include the riskless asset we are dealing with the problem BTNRP2:

$$
\max _{x} \hat{\mu}^{T} x-\epsilon\left\|\Sigma^{1 / 2} x\right\|_{2}+W_{0} R
$$


where $\hat{\mu}=\hat{r}-R e$. The Lagrange dual of the above problem is equivalent to the feasibility problem $z=-\Sigma^{-1 / 2} \hat{\mu},\|z\|_{2} \leq \epsilon$. I.e. by the conic duality theorem, the primal problem is solvable if $\left\|\Sigma^{-1 / 2} \hat{\mu}\right\|_{2}<\epsilon$ in which case the primal optimal solution is the origin. If $\left\|\Sigma^{-1 / 2} \hat{\mu}\right\|_{2}>\epsilon$ then the primal is unbounded since it is trivially feasible. In fact, when solving adjustable robust dynamic version of RMVP3 backwards, one immediately encounters a problem of the type BTNRP2, and the process stalls.

\section{The adjustable robust dynamic Ben-Tal-Nemirovski portfolios}

Let us now consider the portfolio selection problem of the previous section in multiple periods. For ease of exposition, it suffices to examine the case of two periods. As previously, we have two time points $n=0,1$ at which the portfolio choice is made, the end of the time horizon $n=2$ (in general $n=N)$ is the moment where the final realized portfolio value, say $W_{2}\left(W_{N}\right)$ is revealed. The $m$ risky assets have independent uncertain returns in each time period, i.e. the return vector $r_{1}$ is uncertain around the nominal value $\hat{r}_{1}$ with positive definite (variance-covariance) matrix $\Sigma_{1}$, and similar statements hold for mean vector $r_{2}$ with nominal return $\hat{r}_{2}$ and positive definite (variancecovariance) matrix $\Sigma_{2}$. We confine the return $r_{1}$ of the first period to take values in the ellipsoidal ambiguity set around $\hat{r}_{1}: U_{r}^{1}=\left\{r_{1} \mid\left\|\Sigma_{1}^{-1 / 2}\left(r_{1}-\hat{r}_{1}\right)\right\|_{2} \leq \epsilon_{1}\right\}$, and the return $r_{2}$ of the second period to take values in the ellipsoidal ambiguity set around $\hat{r}_{2}: U_{r}^{2}=\left\{r_{2} \mid\left\|\Sigma_{2}^{-1 / 2}\left(r_{2}-\hat{r}_{2}\right)\right\|_{2} \leq \epsilon_{2}\right\}$. The adjustable robustness framework now consists in making the dynamic portfolio choice according to the solution of the following problem:

$$
V_{2}=\max _{x_{2} \in X_{2}} \min _{r_{2} \in U_{r}^{2}} r_{2}^{T} x_{2}
$$

and

$$
V_{1}=\max _{x_{1} \in X_{1}} \min _{r_{1} \in U_{r}^{1}} V_{2}
$$

where $X_{1}=\left\{x \in \mathbb{R}^{m} \mid e^{T} x_{1}=W_{0}\right\}$ and $X_{2}=\left\{x \in \mathbb{R}^{m} \mid e^{T} x_{2}=W_{1}\right\}$ where we use $W_{1}$ to represent the portfolio value at the end of period 1, i.e. at time point $n=1$ where the investor is about to make the second-period portfolio decision. Let us solve the problem backwards again. To compute $V_{2}$ using Proposition 5, we have the solution $x_{2}^{*}=\frac{W_{1}}{\sqrt{\Delta_{2}}} \Sigma_{2}^{-1}\left(\hat{r}_{2}-\frac{B_{2}-\sqrt{\Delta_{2}}}{C_{2}} e\right)$ where $A_{2}=\hat{r}_{2}^{T} \Sigma_{2}^{-1} \hat{r}_{2}, B_{2}=$ $e^{T} \Sigma_{2}^{-1} \hat{r}_{2}, C_{2}=e^{T} \Sigma_{2}^{-1} e, \Delta_{2}=B_{2}^{2}-A_{2} C_{2}+C_{2} \epsilon_{2}^{2}$ provided that $\epsilon_{2}>\sqrt{A_{2}-\frac{B_{2}^{2}}{C_{2}}}$. After some simple algebra, we compute $V_{2}$ to be $V_{2}=W_{1}\left(\frac{\kappa_{2}-\epsilon_{2} \phi_{2}}{\sqrt{\Delta_{2}}}\right)$ where

$$
\kappa_{2}=A_{2}-\frac{B_{2}^{2}}{C_{2}}+\frac{B_{2} \sqrt{\Delta_{2}}}{C_{2}}, \phi_{2}=\sqrt{A_{2}-\frac{B_{2}^{2}}{C_{2}}+\frac{\Delta_{2}}{C_{2}}} .
$$

Since $W_{1}=r_{1}^{T} x_{1}$ we have the problem $V_{1}=\max _{x_{1} \in X_{1}} \min _{r_{1} \in U_{r}^{1}}\left(\frac{\kappa_{2}-\epsilon_{2} \phi_{2}}{\sqrt{\Delta_{2}}}\right) r_{1}^{T} x_{1}$, which is exactly: $V_{1}=\max _{x_{1} \in X_{1}}\left(\frac{\kappa_{2}-\epsilon_{2} \phi_{2}}{\sqrt{\Delta_{2}}}\right)\left(\hat{r}_{1}^{T} x_{1}-\epsilon_{1}\left\|\Sigma_{1}^{1 / 2} x_{1}\right\|_{2}\right)$. The above problem is convex provided that the constant $\frac{\kappa_{2}-\epsilon_{2} \phi_{2}}{\sqrt{\Delta_{2}}}$ is positive, which is equivalent to ask that $\epsilon_{2}<\frac{\kappa_{2}}{\phi_{2}}$. Assuming this condition fulfilled,

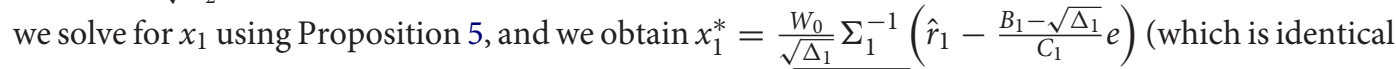
to $x_{2}^{*}$ after the necessary changes) provided that $\epsilon_{1}>\sqrt{A_{1}-\frac{B_{1}^{2}}{C_{1}}}$ and $A_{1}, B_{1}, C_{1}$ and $\Delta_{1}$ are defined analogously.

When the above solution process is extended to $N>2$ periods with the required definitions of $\hat{r}_{n}$, positive definite $\Sigma_{n}$, sets $U_{r}^{n}$, and positive $\epsilon_{n}$, we have the following result. 
Proposition 6: The adjustable robust dynamic portfolio policy of an BTNRP investor with uncertainty radii $\epsilon_{n}, n=1, \ldots, N$ and initial wealth $W_{0}$, defined as the solution to the following problem:

$$
\begin{gathered}
V_{N}=\max _{x_{N} \in X_{N}} \min _{r_{N} \in U_{r}^{N}} r_{N}^{T} x \\
V_{N-1}=\max _{x_{N-1} \in X_{N-1}} \min _{r_{N-1} \in U_{r}^{N-1}} V_{N} \\
\vdots \\
V_{1}=\max _{x_{1} \in X_{1}} \min _{r_{1} \in U_{r}^{1}} V_{2},
\end{gathered}
$$

is given by

$$
x_{n}^{*}=\frac{W_{n-1}}{\sqrt{\Delta_{n}}} \Sigma_{n}^{-1}\left(\hat{r}_{n}-\frac{B_{n}-\sqrt{\Delta_{n}}}{C_{n}} e\right), n=1, \ldots, N,
$$

where

$$
A_{n}=\hat{r}_{n}^{T} \Sigma_{n}^{-1} \hat{r}_{n}, B_{n}=e^{T} \Sigma_{n}^{-1} \hat{r}_{n}, C_{n}=e^{T} \Sigma_{n}^{-1} e, \Delta_{n}=B_{n}^{2}-A_{n} C_{n}+C_{n} \epsilon_{n}^{2}
$$

provided that $\epsilon_{n}>\sqrt{A_{n}-\frac{B_{n}^{2}}{C_{n}}}$ for all $n=1, \ldots, N$ and $\epsilon_{n}<\frac{\kappa_{n}}{\phi_{n}}$ for $n=2, \ldots, N$ with

$$
\kappa_{n}=A_{n}-\frac{B_{n}^{2}}{C_{n}}+\frac{B_{n} \sqrt{\Delta_{n}}}{C_{n}}, \phi_{2}=\sqrt{A_{n}-\frac{B_{n}^{2}}{C_{n}}+\frac{\Delta_{n}}{C_{n}}} .
$$

The values $V_{n}, n=1, \ldots, N$ are given by $V_{n}=W_{n-1} \prod_{\ell=n}^{N} \frac{\kappa_{\ell}-\epsilon_{\ell} \phi_{\ell}}{\sqrt{\Delta_{\ell}}}$.

Hence, the BTNRP investor's dynamic adjustable robust portfolio policy is also a myopic policy.

\section{Conclusions}

We presented closed-form portfolio rules for robust MV portfolio optimization problems where short sales (and/or borrowing) restrictions are not taken into account. Without such restrictions, we were able to solve the resulting conic optimization problems analytically, and to extend the rules to dynamic portfolio choice under the adjustable robustness approach using an independence assumption of returns between consecutive periods, where closed-form portfolio rules are rare (with the exception of e.g. [14]).

\section{Note}

1. Garlappi et al. [18] treat the problem RMVP3 that we address further below without a riskless asset. Their solution requires the numerical solution of a quartic polynomial.

\section{Disclosure statement}

No potential conflict of interest was reported by the authors.

\section{References}

[1] Markovitz H. Portfolio selection: efficient diversification of investment. New York (NY): Wiley; 1959.

[2] Best MJ. Portfolio optimization. Financial mathematics series. Boca Raton (FL): Chapman \& Hall/CRC; 2010.

[3] Ingersoll J. Theory of financial decision making. Savage (MD): Rowman-Littlefield; 1987.

[4] Ross S. An introduction to mathematical finance: options and other topics. Cambridge: Cambridge University Press; 1999.

[5] Steinbach M. Markowitz revisited: mean-variance models in financial portfolio analysis. SIAM Rev. 2001;43: 31-85. 
[6] Ben-Tal A, Nemirovski A. Robust solutions to uncertain linear programming problems. Oper. Res. Lett. 1999;25:1-13.

[7] Ben-Tal A, Nemirovski A. Robust convex optimization. Math. Oper. Res. 1998;23:769-805.

[8] Ben-Tal A, Nemirovski A. Lectures on modern convex optimization. Philadelphia: SIAM-MPS series on optimization; 2001.

[9] Bertsimas D, Brown DB, Caramanis C. Theory and applications of robust optimization. SIAM Rev. 2011;53: 464-501.

[10] Fabozzi FJ, Kolm PN, Pachamanova DA, et al. Robust portfolio optimization and management. New York (NY): John Wiley \& Sons; 2007.

[11] Best MJ, Grauer R. Sensitivity analysis for mean-variance portfolio problems. Manage. Sci. 1991;37:980-989.

[12] Best MJ, Grauer RR. On the sensitivity of mean-variance-efficient portfolios to changes in asset means: some analytical and computational results. Rev. Financial Stud. 1991;4:315-342.

[13] Black F, Litterman R. Global portfolio optimization, Financial Anal. J. 1992;48:28-43.

[14] Chen L, He S, Zhang Sh. Tight bounds for some risk measures, with applications to robust portfolio selection. Oper. Res. 2011;59:847-865.

[15] Delage E, Ye Y. Distributionally robust optimization under moment uncertainty with application to data-driven problems. Oper. Res. 2010;58:596-612.

[16] DeMiguel V, Nogales F. Portfolio selection with robust estimation. Oper. Res. 2009;57:560-577.

[17] Fabozzi FJ, Huang D, Zhou G. Robust portfolios: contributions from operations research and finance. Ann. Oper. Res. 2010;176:191-220.

[18] Garlappi L, Uppal R, Wang T. Portfolio selection with parameter and model uncertainty: a multi-prior approach. Rev. Financial Stud. 2007;20:41-81.

[19] Goldfarb D, Iyengar G. Robust portfolio selection problems. Math. Oper. Res. 2003;28:1-38.

[20] Popescu I. Robust mean-covariance solutions for stochastic optimization. Oper. Res. 2005;55:98-112.

[21] Ben-Tal A, Goryashko A, Guslitzer E, et al. Adjustable robust solutions to uncertain linear programs. Math. Program. 2004;99:351-376.

[22] Pınar MÇ, Tütüncü R. Robust profit opportunities in risky financial portfolios. Oper. Res. Lett. 2005;33:331-340.

[23] Takeda A, Taguchi S, Tütüncü R. Adjustable robust optimization models for a non-linear two-period system. J. Optim. Theory Appl. 2008;136:275-295.

[24] Bertsekas DP. Dynamic programming and stochastic control. New York (NY): Academic Press; 1976.

[25] Li D, Ng W-L. Optimal dynamic portfolio selection: multiperiod mean-variance formulation. Math. Finance. 2000;10:387-406. 